\title{
DENSITY ESTIMATES ON COMPOSITE POLYNOMIALS
}

\author{
WAI SHUN CHEUNG, TUEN WAI NG ${ }^{\circledR}$ and CHIU YIN TSANG \\ (Received 9 January 2013; accepted 14 May 2013; first published online 7 August 2013) \\ Communicated by P. Fenton
}

\begin{abstract}
Ritt introduced the concepts of prime and composite polynomials and proved three fundamental theorems on factorizations (in the sense of compositions) of polynomials in 1922. In this paper, we shall give a density estimate on the set of composite polynomials.
\end{abstract}

2010 Mathematics subject classification: primary 30C10; secondary 15A60.

Keywords and phrases: prime and composite polynomials, density estimates.

\section{Introduction and preliminaries}

Let $p$ be a nonlinear polynomial in one complex variable. We say that $p$ is prime if and only if there do not exist two complex polynomials $q_{1}$ and $q_{2}$ both with degree greater than one such that $p(z)=q_{1}\left(q_{2}(z)\right)$. Otherwise, $p$ is called composite or decomposable.

Clearly, for a given polynomial $p$, one can always factorize it as a composition of prime polynomials only and this factorization will be called a prime factorization. In 1922, Ritt [13] proved three fundamental results on the factorizations of complex polynomials. Since then many people have tried to give different proofs or generalizations of Ritt's theorems to certain classes of rational functions (see, for example, $[2,5,6,8-11,16])$.

It is worth pointing out that the factorizations of entire or meromorphic functions have also been considered by many people. For a detailed discussion of this topic, we refer the reader to $[3,4,7]$. One can also find a discussion on factorizations of infinite Blaschke products in [15].

The set of critical values of a polynomial plays an important role in determining if the polynomial is prime or not (see, for example, Theorem A below). By considering the number of distinct critical values of a polynomial, Beardon [1] showed that for

The second author was partially supported by RGC grant HKU 704611P. The third author was partially supported by graduate studentship of HKU and RGC grant HKU 704409P and HKU 704611P.

(c) 2013 Australian Mathematical Publishing Association Inc. 1446-7887/2013 \$16.00 
each fixed positive integer $n$, the set of degree $n$ composite polynomials lies in some hypersurface in $\mathbb{C}^{n+1}$ which implies that the set of composite polynomials is of measure zero and hence almost all polynomials are prime. In this paper, we shall give a density estimate on how small the set of degree $n$ composite polynomials is. This kind of density estimation was first used by Smale in his work on the efficiency of Newton's method [14]. In fact, Smale found a density estimate on a set $\mathcal{V}_{\rho, n}$ of 'bad' polynomials of degree $n$ which fail to arrive at an approximate zero when applying Newton's method a certain fixed number of times. Here, a point $z_{0}$ is called an approximate zero of $p$ if $z_{0} \rightarrow z^{*}, p\left(z^{*}\right)=0$ and $\left|p\left(z_{n}\right) / p\left(z_{n-1}\right)\right|<1 / 2$ for all $n \in \mathbb{N}$, where $z_{n+1}=z_{n}-\left(p\left(z_{n}\right) / p^{\prime}\left(z_{n}\right)\right)$. See Remark 2.2 in Section 2 for the details of Smale's density estimate on $\mathcal{V}_{\rho, n}$.

\section{The main result}

Without loss of generality, we may assume that $p$ is a normalized polynomial of degree $n \geq 2$, that is, $p(z)=z^{n}+a_{n-1} z^{n-1}+\cdots+a_{1} z$. Now $w$ is a critical value of $p$ if and only if $p^{\prime}(z)=0$ and $p(z)-w=0$ have a common root if and only if the resultant $\operatorname{Res}\left(p-w, p^{\prime}\right)=0$. Denote $\operatorname{Res}\left(p-w, p^{\prime}\right)$ by $\Phi(w)$. Clearly, $\Phi(w)$ is a polynomial in $w$ of degree $n-1$ and $p$ has $n-1$ critical values (which may not be distinct). Now we state the theorem proved by Beardon [1].

Theorem A [1, Theorem 3.2]. If a polynomial $p$ of degree $n \geq 2$ has more than $\lfloor n / 2\rfloor$ distinct critical values (here $\lfloor x\rfloor$ is the integer part of a real number $x$ ), then it is prime. In particular, if p has $n-1$ distinct critical values, then it is prime.

If $p$ is composite, then $p$ has at most $n-2$ distinct critical values by Theorem $A$, and this is equivalent to saying that $\Phi(w)=0$ has a repeated root or equivalently,

$$
\Psi\left(a_{1}, \ldots, a_{n-1}\right):=\operatorname{Res}\left(\Phi, \Phi^{\prime}\right)=0
$$

Let $\mathcal{W}_{n}=\left\{\left(a_{1}, \ldots, a_{n-1}\right) \in \mathbb{C}^{n-1}: \Psi\left(a_{1}, \ldots, a_{n-1}\right)=0\right\}$. Then the set $C_{n}:=\left\{\left(a_{1}\right.\right.$, $\left.\ldots, a_{n-1}\right) \in \mathbb{C}^{n-1}: z^{n}+a_{n-1} z^{n-1}+\cdots+a_{1} z$ is composite $\}$ is contained in $\mathcal{W}_{n}$.

Now we are going to obtain a density estimate on $C_{n}$. Let $P_{n}$ be the set of normalized polynomials of degree $n$, that is, $P_{n}=\left\{p: p(z)=z^{n}+a_{n-1} z^{n-1}+\cdots+a_{1} z, a_{i} \in \mathbb{C}\right\}$. Thus $P_{n}$ can be identified with $\mathbb{C}^{n-1}=\left\{\left(a_{1}, \ldots, a_{n-1}\right): a_{i} \in \mathbb{C}\right\}$. Let $P(R)$ be the polycylinder defined by $\left\{\mathbf{a}=\left(a_{1}, \ldots, a_{n-1}\right) \in P_{n}:\left|a_{i}\right|<R, i=1, \ldots, n-1\right\}$. To obtain the volume of $P(R)$, we consider the standard volume on $\mathbb{C}^{n-1}=\mathbb{R}^{2 n-2}$ for $P_{n}$. Let $a_{j}=x_{j}+i y_{j}$ and $D_{j}(R)=\left\{\left(x_{j}, y_{j}\right) \in \mathbb{R}^{2}: x_{j}^{2}+y_{j}^{2}<R^{2}\right\}$. Then

$$
\begin{aligned}
\operatorname{Vol}(P(R)) & =\int_{P(R)} d \mathbf{a}=\int_{\left|a_{n-1}\right|<R} \cdots \int_{\left|a_{1}\right|<R} d a_{1} \cdots d a_{n-1} \\
& =\left(\int_{\left|a_{1}\right|<R} d a_{1}\right) \cdots\left(\int_{\left|a_{n-1}\right|<R} d a_{n-1}\right)
\end{aligned}
$$




$$
\begin{aligned}
& =\left(\int_{D_{1}(R)} d x_{1} d y_{1}\right) \cdots\left(\int_{D_{n-1}(R)} d x_{n-1} d y_{n-1}\right) \\
& =\left(\pi R^{2}\right)^{n-1} .
\end{aligned}
$$

Let $\mathcal{S}$ be any subset of $\mathcal{W}_{n}$, let $\rho$ be any positive real number, and define

$$
U_{\rho}(\mathcal{S})=\bigcup_{f_{0} \in \mathcal{S}} U_{\rho}\left(f_{0}\right)
$$

where $U_{\rho}\left(f_{0}\right)=\left\{f \in P_{n}:\left|f^{\prime}(0)-f_{0}^{\prime}(0)\right|<\rho, f^{\prime \prime}(z)=f_{0}^{\prime \prime}(z)\right.$ for all $\left.z\right\}$. Clearly, $U_{\rho}\left(C_{n}\right) \subset$ $U_{\rho}\left(\mathcal{W}_{n}\right)$. Now we can state our main result.

Theorem 2.1. For any $R>\rho>0$,

$$
\frac{\operatorname{Vol}\left(U_{\rho}\left(C_{n}\right) \cap P(R)\right)}{\operatorname{Vol}(P(R))} \leq \frac{\operatorname{Vol}\left(U_{\rho}\left(\mathcal{W}_{n}\right) \cap P(R)\right)}{\operatorname{Vol}(P(R))} \leq \frac{n(n-2) \rho^{2}}{R^{2}}
$$

REMARK 2.2. One should compare Theorem 2.1 with

Smale's Density Estimate [14, Theorem 5.(1)]. For any $R>\frac{1}{3}$,

$$
\frac{\operatorname{Vol}\left(\mathcal{V}_{\rho, n} \cap P(R)\right)}{\operatorname{Vol}(P(R))} \leq 150(n+2)^{4 / 3} \rho^{2 / 3},
$$

where $\mathcal{V}_{\rho, n}$ is mentioned in Section 1.

By considering the exponents of $\rho$ in (2.1) and (2.2), for a fixed positive integer $n$, the upper bound in the estimate in Theorem 2.1 is much smaller than the one in Smale's estimate for sufficiently small $\rho>0$.

REMARK 2.3. We shall see in Section 3 that the constant $n(n-2) / R^{2}$ in the estimate

$$
\frac{\operatorname{Vol}\left(U_{\rho}\left(C_{n}\right) \cap P(R)\right)}{\operatorname{Vol}(P(R))} \leq \frac{n(n-2) \rho^{2}}{R^{2}}
$$

is far from being sharp because $C_{n}$ is in general a small subset of $\mathcal{W}_{n}$.

To prove Theorem 2.1, we need the following lemma.

Lemma 2.4. The subset $\mathcal{W}_{n} \subset P_{n}$ is a complex algebraic hypersurface defined by the polynomial equation $\Psi\left(a_{1}, \ldots, a_{n-1}\right)=0$, where $\Psi$ is a polynomial of degree $n(n-2)$ in $a_{1}$.

Proof of Lemma 2.4. Let us recall the definition of the resultant. For any two polynomials $u(z)=u_{m} z^{m}+u_{m-1} z^{m-1}+\cdots+u_{0}$ and $v(z)=v_{n} z^{n}+\cdots+v_{0}$, the resultant 
$\operatorname{Res}(u(z), v(z))$ of $u$ and $v$ is defined to be the determinant of the following $(m+n) \times$ $(m+n)$ matrix:

$$
\left[\begin{array}{cccccccc}
u_{m} & u_{m-1} & \cdots & u_{1} & u_{0} & 0 & \cdots & 0 \\
0 & u_{m} & \ddots & u_{2} & u_{1} & u_{0} & \cdots & 0 \\
\vdots & \vdots & \ddots & \ddots & \vdots & \vdots & \ddots & \vdots \\
0 & 0 & \cdots & u_{m} & u_{m-1} & u_{m-2} & \cdots & u_{0} \\
v_{n} & v_{n-1} & \cdots & \cdots & v_{0} & 0 & \cdots & 0 \\
0 & v_{n} & \ddots & \cdots & v_{1} & v_{0} & \cdots & 0 \\
\vdots & \vdots & \ddots & \ddots & \vdots & \vdots & \ddots & \vdots \\
0 & 0 & \cdots & v_{n} & v_{n-1} & v_{n-2} & \cdots & v_{0}
\end{array}\right] .
$$

As

$$
p(z)-w=z^{n}+a_{n-1} z^{n-1}+\cdots+a_{1} z-w
$$

and

$$
p^{\prime}(z)=n z^{n-1}+(n-1) a_{n-1} z^{n-2}+\cdots+2 a_{2} z+a_{1},
$$

we can see that $\Phi(w):=\operatorname{Res}\left(p-w, p^{\prime}\right)$ is the determinant of the following $(2 n-1) \times(2 n-1)$ matrix:

$$
\left[\begin{array}{ccccccccc}
1 & a_{n-1} & \cdots & a_{2} & a_{1} & -w & 0 & \cdots & 0 \\
0 & 1 & \ddots & a_{3} & a_{2} & a_{1} & -w & \cdots & 0 \\
\vdots & \vdots & \ddots & \ddots & \vdots & \vdots & \vdots & \ddots & \vdots \\
0 & 0 & \cdots & 1 & a_{n-1} & a_{n-2} & a_{n-3} & \cdots & -w \\
n & (n-1) a_{n-1} & \cdots & 2 a_{2} & a_{1} & 0 & \cdots & \cdots & 0 \\
0 & n & \ddots & 3 a_{3} & 2 a_{2} & a_{1} & 0 & \cdots & 0 \\
\vdots & \vdots & \ddots & \ddots & \vdots & \vdots & \ddots & \ddots & \vdots \\
0 & 0 & \cdots & n & (n-1) a_{n-1} & \cdots & \cdots & a_{1} & 0 \\
0 & 0 & \cdots & 0 & n & (n-1) a_{n-1} & \cdots & \cdots & a_{1}
\end{array}\right] .
$$

Clearly, $\Phi(w)$ is a polynomial in $w$ of degree $n-1$ whose leading coefficient is $(-1)^{n-1} n^{n}$, that is,

$$
\Phi(w)=\sum_{i=0}^{n-1} F_{i}\left(a_{1}, \ldots, a_{n-1}\right) w^{i},
$$

where $F_{n-1}\left(a_{1}, \ldots, a_{n-1}\right)=(-1)^{n-1} n^{n}$.

To find the coefficient of $a_{1}^{n}$ in $F_{0}$, we consider the determinant of the matrix in (2.3). By subtracting the $(n-1+i)$ th row from the $i$ th row for the determinant of the matrix 
in (2.3) $(i=1, \ldots, n-1)$, we can see that $\Phi(w)$ is the determinant of the following $(2 n-1) \times(2 n-1)$ matrix:

$$
\left[\begin{array}{ccccccccc}
1-n & (2-n) a_{n-1} & \cdots & -a_{2} & 0 & -w & 0 & \cdots & 0 \\
0 & 1-n & \ddots & -2 a_{3} & -a_{2} & 0 & -w & \cdots & 0 \\
\vdots & \vdots & \ddots & \ddots & \vdots & \vdots & \vdots & \ddots & \vdots \\
0 & 0 & \cdots & 1-n & (2-n) a_{n-1} & (3-n) a_{n-2} & (4-n) a_{n-3} & \cdots & -w \\
n & (n-1) a_{n-1} & \cdots & 2 a_{2} & a_{1} & 0 & \cdots & \cdots & 0 \\
0 & n & \ddots & 3 a_{3} & 2 a_{2} & a_{1} & 0 & \cdots & 0 \\
\vdots & \vdots & \ddots & \ddots & \vdots & \vdots & \ddots & \ddots & \vdots \\
0 & 0 & \cdots & n & (n-1) a_{n-1} & \cdots & \cdots & a_{1} & 0 \\
0 & 0 & \cdots & 0 & n & (n-1) a_{n-1} & \cdots & \cdots & a_{1}
\end{array}\right] .
$$

It is easily seen that

$$
F_{0}\left(a_{1}, a_{2}, \ldots, a_{n-1}\right)=(-1)^{n-1}(n-1)^{n-1} a_{1}^{n}+\sum_{i=0}^{n-1} G_{i}\left(a_{2}, \ldots, a_{n-1}\right) a_{1}^{i},
$$

where $G_{i}$ is a polynomial in the variables $a_{2}, \ldots, a_{n-1}$.

Now we show that for $i=1, \ldots, n-2$, each term of $F_{i}$ must involve some $a_{j}$ for $j=2, \ldots, n-1$. To prove this, consider

$$
\begin{aligned}
& \left.\Phi\right|_{a_{2}=\cdots=a_{n-1}=0}(w) \\
& =\left|\begin{array}{ccccccccc}
1-n & 0 & \cdots & 0 & 0 & -w & 0 & \cdots & 0 \\
0 & 1-n & \ddots & 0 & 0 & 0 & -w & \cdots & 0 \\
\vdots & \vdots & \ddots & \ddots & \vdots & \vdots & \vdots & \ddots & \vdots \\
0 & 0 & \cdots & 1-n & 0 & 0 & 0 & \cdots & -w \\
n & 0 & \cdots & 0 & a_{1} & 0 & \cdots & \cdots & 0 \\
0 & n & \ddots & 0 & 0 & a_{1} & 0 & \cdots & 0 \\
\vdots & \vdots & \ddots & \ddots & \vdots & \vdots & \ddots & \ddots & \vdots \\
0 & 0 & \cdots & n & 0 & \cdots & \cdots & a_{1} & 0 \\
0 & 0 & \cdots & 0 & n & 0 & \cdots & \cdots & a_{1}
\end{array}\right|_{(2 n-1) \times(2 n-1)} \\
& =a_{1}\left|\begin{array}{cc}
(1-n) I_{n-1} & -w J_{n-1} \\
n I_{n-1} & a_{1} I_{n-1}
\end{array}\right|+(-1)^{n-1} n\left|\begin{array}{cc}
(1-n) I_{n-1} & -w I_{n-1} \\
n I_{n-1} & a_{1} J_{n-1}^{T}
\end{array}\right|,
\end{aligned}
$$

where the last equality holds by expanding the last row of the above determinant and where $I_{m}$ and $J_{m}$ denote the $m \times m$ identity matrix and the $m \times m$ Jordan block with eigenvalues 0 respectively. Using the fact that $\operatorname{det}\left[\begin{array}{ll}A & B \\ C & D\end{array}\right]=\operatorname{det}\left(A D-A C A^{-1} B\right)$ if $A, B, C, D \in \mathbb{C}^{m \times m}$ and $A$ is invertible,

$$
\begin{aligned}
\left.\Phi\right|_{a_{2}}=\cdots & =a_{n-1}=0 \\
& =a_{1} \operatorname{det}\left[a_{1}(1-n) I_{n-1}+w n J_{n-1}\right]+(-1)^{n-1} n \operatorname{det}\left[a_{1}(1-n) J_{n-1}^{T}+w n I_{n-1}\right] \\
& =(1-n)^{n-1} a_{1}^{n}+(-1)^{n-1} w^{n-1} n^{n} .
\end{aligned}
$$


In particular,

$$
F_{j}\left(a_{1}, 0, \ldots, 0\right)=0 \text { for } j=1, \ldots n-2 .
$$

Therefore, for $j=1, \ldots, n-2$,

$$
F_{j}\left(a_{1}, a_{2}, \ldots, a_{n-1}\right)=\sum_{s_{2}+\cdots+s_{n-1} \geq 1} H_{s_{2}, \ldots, s_{n-1}}^{j}\left(a_{1}\right) a_{2}^{s_{2}} \cdots a_{n-1}^{s_{n-1}},
$$

where $H_{s_{2}, \ldots, s_{n-1}}^{j}$ is a polynomial in the variable $a_{1}$.

As

$$
\Phi(w)=F_{n-1} w^{n-1}+\cdots+F_{0}
$$

and

$$
\Phi^{\prime}(w)=(n-1) F_{n-1} w^{n-2}+(n-2) F_{n-2} w^{n-3}+\cdots+F_{1},
$$

it follows that $\Psi\left(a_{1}, \ldots, a_{n-1}\right)$ is the determinant of the following $(2 n-3) \times(2 n-3)$ matrix

$$
\left[\begin{array}{ccccccccc}
F_{n-1} & F_{n-2} & \cdots & F_{2} & F_{1} & F_{0} & 0 & \cdots & 0 \\
0 & F_{n-1} & \ddots & F_{3} & F_{2} & F_{1} & F_{0} & \cdots & 0 \\
\vdots & \vdots & \ddots & \ddots & \vdots & \vdots & \vdots & \ddots & \vdots \\
0 & 0 & \cdots & F_{n-1} & F_{n-2} & F_{n-3} & F_{n-4} & \cdots & F_{0} \\
(n-1) F_{n-1} & (n-2) F_{n-2} & \cdots & 2 F_{2} & F_{1} & 0 & \cdots & \cdots & 0 \\
0 & (n-1) F_{n-1} & \ddots & 3 F_{3} & 2 F_{2} & F_{1} & 0 & \cdots & 0 \\
\vdots & \vdots & \ddots & \ddots & \vdots & \vdots & \ddots & \ddots & \vdots \\
0 & 0 & \cdots & (n-1) F_{n-1} & (n-2) F_{n-2} & \cdots & \cdots & F_{1} & 0 \\
0 & 0 & \cdots & 0 & (n-1) F_{n-1} & (n-2) F_{n-2} & \cdots & \cdots & F_{1}
\end{array}\right] .
$$

Therefore,

$$
\begin{aligned}
\Psi\left(a_{1}, \ldots, a_{n-1}\right)= & (n-1)^{n-1} F_{n-1}^{n-1} F_{0}^{n-2} \\
& +\sum_{r_{1}+\cdots+r_{n-2} \geq 1} P_{r_{1}, \ldots, r_{n-2}}\left(F_{0}, F_{n-1}\right) F_{1}^{r_{1}} \cdots F_{n-2}^{r_{n-2}},
\end{aligned}
$$

where $P_{r_{1}, \ldots, r_{n-2}}$ is a polynomial in the variables $F_{0}, F_{n-1}$. By (2.4) and (2.5), the first term in (2.6) is

$$
(n-1)^{n-1} F_{n-1}^{n-1} F_{0}^{n-2}=(-1)^{n-1}(n-1)^{(n-1)^{2}} n^{n(n-1)} a_{1}^{n(n-2)}+\sum_{i=0}^{n(n-2)-1} T_{i}\left(a_{2}, \ldots, a_{n-1}\right) a_{1}^{i}
$$

and the second term in (2.6) is

$$
\sum_{r_{1}+\cdots+r_{n-2} \geq 1} P_{r_{1}, \ldots, r_{n-2}}\left(F_{0}, F_{n-1}\right) F_{1}^{r_{1}} \cdots F_{n-2}^{r_{n-2}}=\sum_{t_{2}+\cdots+t_{n-1} \geq 1} Q_{t_{2}, \ldots, t_{n-1}}\left(a_{1}\right) a_{2}^{t_{2}} \cdots a_{n-1}^{t_{n-1}},
$$


where $T_{i}$ is a polynomial in the variables $a_{2}, \ldots, a_{n-1}$ and $Q_{t_{2}, \ldots, t_{n-1}}$ is a polynomial in $a_{1}$ only. Then

$$
\begin{gathered}
\Psi\left(a_{1}, \ldots, a_{n-1}\right)=(-1)^{n-1}(n-1)^{(n-1)^{2}} n^{n(n-1)} a_{1}^{n(n-2)}+\sum_{i=0}^{n(n-2)-1} T_{i}\left(a_{2}, \ldots, a_{n-1}\right) a_{1}^{i} \\
+\sum_{t_{2}+\cdots+t_{n-1} \geq 1} Q_{t_{2}, \ldots, t_{n-1}}\left(a_{1}\right) a_{2}^{t_{2}} \cdots a_{n-1}^{t_{n-1}} .
\end{gathered}
$$

In particular, we can see that $\Psi$ has degree at least $n(n-2)$ in $a_{1}$.

On the other hand, by expressing $\Psi$ in terms of the zeros $w_{1}, \ldots, w_{n-1}$ of $\Phi(w)$, that is,

$$
\Psi\left(a_{1}, \ldots, a_{n-1}\right)=F_{n-1}^{2 n-4} \prod_{i<j}\left(w_{i}-w_{j}\right)^{2},
$$

we can show that $\Psi$ has degree at most $n(n-2)$ in $a_{1}$. This suffices to show that for any fixed $a_{2}, \ldots, a_{n-1}$,

$$
\Psi_{a_{2}, \ldots, a_{n-1}}\left(a_{1}\right)=\Psi\left(a_{1}, \ldots, a_{n-1}\right) \leq O\left(\left|a_{1}\right|^{n(n-2)}\right) .
$$

To prove this, we need to use a theorem which gives an upper bound for the zeros of a polynomial in terms of the coefficients of the polynomial. To state this result, we need the following definition.

Definition 2.5 [12, Definition 8.1.2]. Let $f(z)=c_{0}+c_{1} z+\cdots+c_{n} z^{n}$ be a polynomial of degree $n \geq 1$. Then the Cauchy bound of $f$, denoted by $\rho[f]$, is defined as the unique positive root of the equation $\left|c_{0}\right|+\left|c_{1}\right| x+\cdots+\left|c_{n-1}\right| x^{n-1}=\left|c_{n}\right| x^{n}$ when $f$ is not a monomial, and as zero otherwise (the uniqueness of the root was proved in [12, Lemma 8.1.1]).

Theorem B [12, Corollary 8.1.8]. If $f(z)=c_{0}+c_{1} z+\cdots+c_{n} z^{n}$, where $c_{n} \neq 0$, then

$$
\rho[f] \leq \max _{0 \leq v \leq n-1}\left(n\left|\frac{c_{v}}{c_{n}}\right|\right)^{1 /(n-v)} .
$$

REMARK 2.6. Notice that all the zeros of the nonconstant polynomial $f$ lie in the closed disk with centre at the origin and radius $\rho[f]$ (see [12, Theorem 8.1.3]).

Let $r_{i}(i=1, \ldots, n-1)$ be the zeros (which may not be distinct) of $p^{\prime}$. Applying Theorem B to $f(z)=p^{\prime}(z)=n z^{n-1}+(n-1) a_{n-1} z^{n-2}+\cdots+2 a_{2} z+a_{1}$ for any fixed $a_{2}, \ldots, a_{n}$, then

$$
\left|r_{i}\right| \leq \rho\left[p^{\prime}\right] \leq \max _{0 \leq v \leq n-2}\left(\frac{(v+1)(n-1)}{n}\left|a_{v+1}\right|\right)^{1 /((n-1)-v)} \leq O\left(\left|a_{1}\right|^{1 /(n-1)}\right) .
$$

Note that $p\left(r_{i}\right)$ is a critical value of $p$ and therefore $\Phi(w)=0$ if and only if $w=p\left(r_{i}\right)$. Hence

$$
\Psi\left(a_{1}, \ldots, a_{n-1}\right)=F_{n-1}^{2 n-4} \prod_{i<j}\left(p\left(r_{i}\right)-p\left(r_{j}\right)\right)^{2}=n^{2 n(n-2)} \prod_{i<j}\left(p\left(r_{i}\right)-p\left(r_{j}\right)\right)^{2} .
$$


As

$$
\left(p\left(r_{i}\right)-p\left(r_{j}\right)\right)^{2} \leq O\left(\left|a_{1}\right|^{2 n /(n-1)}\right)
$$

and there are exactly $(n-1)(n-2) / 2$ distinct pairs of $p\left(r_{i}\right)-p\left(r_{j}\right)$ for $i<j$,

$$
\begin{aligned}
\Psi_{a_{2}, \ldots, a_{n-1}}\left(a_{1}\right) & =\Psi\left(a_{1}, \ldots, a_{n-1}\right) \\
& =n^{2 n(n-2)} \prod_{i<j}\left(p\left(r_{i}\right)-p\left(r_{j}\right)\right)^{2} \leq O\left(\left|a_{1}\right|^{n(n-2)}\right),
\end{aligned}
$$

for any fixed $a_{2}, \ldots, a_{n}$.

By (2.7) and (2.8), $\Psi$ has degree $n(n-2)$ in $a_{1}$; more precisely, there exist polynomials $R_{0}, \ldots, R_{n(n-2)} \in \mathbb{C}\left[a_{2}, \ldots, a_{n-1}\right]$ such that

$$
\Psi\left(a_{1}, \ldots, a_{n-1}\right)=\sum_{i=0}^{n(n-2)} R_{i}\left(a_{2}, \ldots, a_{n-1}\right) a_{1}^{i},
$$

where $R_{n(n-2)} \not \equiv 0$. Therefore, $\mathcal{W}_{n}$ is the complex hypersurface defined by the polynomial equation $\Psi=0$, where $\Psi$ is of degree $n(n-2)$ in $a_{1}$. This completes the proof of Lemma 2.4 .

Now we are ready to prove Theorem 2.1.

Proof of Theorem 2.1. Let $\chi: \mathbb{C}^{n-1} \rightarrow\{0,1\}$ be the characteristic function of $U_{\rho}\left(\mathcal{W}_{n}\right)$. By Lemma 2.4, we observe that for a generic $\left(a_{2}, \ldots, a_{n-1}\right) \in \mathbb{C}^{n-2}$, the intersection of $\mathcal{W}_{n}$ with the one-dimensional coordinate plane $\left\{\left(z, a_{2}, \ldots, a_{n-1}\right): z \in \mathbb{C}\right\}$ consists of at most $n(n-2)$ points. Hence

$$
\begin{aligned}
\left|\int_{\left|a_{1}\right|<R} \chi\left(a_{1}, a_{2}, \ldots, a_{n-1}\right) d a_{1}\right| & \leq\left|\int_{\left|a_{1}\right|<\infty} \chi\left(a_{1}, a_{2}, \ldots, a_{n-1}\right) d a_{1}\right| \\
& \leq n(n-2) \pi \rho^{2} .
\end{aligned}
$$

By Fubini's theorem,

$$
\begin{aligned}
\frac{\operatorname{Vol}\left(U_{\rho}\left(\mathcal{W}_{n}\right) \cap P(R)\right)}{\operatorname{Vol}(P(R))} & =\frac{1}{\left(\pi R^{2}\right)^{n-1}} \int_{P(R)} \chi(\mathbf{a}) d \mathbf{a} \\
& =\frac{1}{\left(\pi R^{2}\right)^{n-1}} \int_{\left|a_{2}\right|, \ldots,\left|a_{n-1}\right|<R}\left[\int_{\left|a_{1}\right|<R} \chi\left(a_{1}, \ldots, a_{n-1}\right) d a_{1}\right] \\
& \leq \frac{1}{\left(\pi R^{2}\right)^{n-1}} \int_{\left|a_{2}\right|, \ldots,\left|a_{n-1}\right|<R}\left[n(n-2) \pi \rho^{2}\right] d a_{2} \cdots d a_{n-1} \\
& =\frac{1}{\left(\pi R^{2}\right)^{n-1}}\left[n(n-2) \pi \rho^{2}\right]\left(\pi R^{2}\right)^{n-2}=\frac{n(n-2) \rho^{2}}{R^{2}} .
\end{aligned}
$$

Since, $U_{\rho}\left(C_{n}\right) \subset U_{\rho}\left(\mathcal{W}_{n}\right)$,

$$
\frac{\operatorname{Vol}\left(U_{\rho}\left(C_{n}\right) \cap P(R)\right)}{\operatorname{Vol}(P(R))} \leq \frac{\operatorname{Vol}\left(U_{\rho}\left(\mathcal{W}_{n}\right) \cap P(R)\right)}{\operatorname{Vol}(P(R))} \leq \frac{n(n-2) \rho^{2}}{R^{2}} .
$$




\section{Composite polynomials of small degrees}

3.1. Degree four polynomials. By considering composite polynomials of degree four, we shall see that the density estimate of $C_{4}$ in Theorem 2.1 is not sharp. In fact, by Theorem 2.1,

$$
\frac{\operatorname{Vol}\left(U_{\rho}\left(C_{4}\right) \cap P(R)\right)}{\operatorname{Vol}(P(R))} \leq \frac{8 \rho^{2}}{R^{2}} .
$$

However, we actually have the following proposition which gives a better upper bound for the density of $C_{4}$.

Proposition 3.1.

$$
\frac{\operatorname{Vol}\left(U_{\rho}\left(C_{4}\right) \cap P(R)\right)}{\operatorname{Vol}(P(R))} \leq \frac{\rho^{2}}{R^{2}}
$$

To see this, note that if $p(z)=z^{4}+a_{3} z^{3}+a_{2} z^{2}+a_{1} z$ is composite, then $\Psi\left(a_{1}, a_{2}, a_{3}\right)=0$. By using mathematical software such as Mathematica,

$$
\begin{aligned}
\Psi\left(a_{1}, a_{2}, a_{3}\right)=- & 4096\left(a_{3}^{3}-4 a_{3} a_{2}+8 a_{1}\right)^{2} \\
& \times\left(108 a_{1}^{2}-108 a_{3} a_{2} a_{1}+27 a_{3}^{3} a_{1}+32 a_{2}^{3}-9 a_{3}^{2} a_{2}^{2}\right)^{3} .
\end{aligned}
$$

From the proof of Theorem 2.1, we know that the upper bound $8 \rho^{2} / R^{2}$ comes from the fact that $\Psi\left(a_{1}, a_{2}, a_{3}\right)$ is of degree eight in $a_{1}$. We can get a much better bound $\rho^{2} / R^{2}$ by showing that $p(z)$ is composite if and only if $a_{3}^{3}-4 a_{3} a_{2}+8 a_{1}=0$.

To prove this, suppose that $p$ is composite, then there exist some $A, B \in \mathbb{C}$ such that

$$
z^{4}+a_{3} z^{3}+a_{2} z^{2}+a_{1} z=\left(z^{2}+A z\right) \circ\left(z^{2}+B z\right)=z^{4}+2 B z^{3}+\left(A+B^{2}\right) z^{2}+A B z .
$$

Comparing the coefficients, we have $a_{3}=2 B, a_{2}=A+B^{2}, a_{1}=A B$. After elimination of $A$ and $B$, we have $a_{3}^{3}-4 a_{3} a_{2}+8 a_{1}=0$.

Conversely, suppose that $a_{3}^{3}-4 a_{3} a_{2}+8 a_{1}=0$. Then

$$
\begin{aligned}
\left(z^{2}+\left(a_{2}-\frac{a_{3}^{2}}{4}\right) z\right) \circ\left(z^{2}+\frac{a_{3}}{2} z\right) & =z^{4}+a_{3} z^{3}+a_{2} z^{2}+\left(a_{2}-\frac{a_{3}^{2}}{4}\right)\left(\frac{a_{3}}{2}\right) z \\
& =z^{4}+a_{3} z^{3}+a_{2} z^{2}+a_{1} z .
\end{aligned}
$$

So $p$ is composite. Hence $C_{4}=\left\{\left(a_{1}, a_{2}, a_{3}\right) \in \mathbb{C}^{3}: a_{3}^{3}-4 a_{3} a_{2}+8 a_{1}=0\right\}$.

Proof of Proposition 3.1. Note that $a_{3}^{3}-4 a_{3} a_{2}+8 a_{1}$ is of degree one in $a_{1}$, hence for any $\left(a_{2}, a_{3}\right) \in \mathbb{C}^{2}$, the intersection of $C_{4}$ with the one-dimensional coordinate plane $\left\{\left(z, a_{2}, a_{3}\right): z \in \mathbb{C}\right\}$ consists of exactly one point. Hence

$$
\left|\int_{\left|a_{1}\right|<R} \chi\left(a_{1}, a_{2}, a_{3}\right) d a_{1}\right| \leq\left|\int_{\left|a_{1}\right|<\infty} \chi\left(a_{1}, a_{2}, a_{3}\right) d a_{1}\right|=\pi \rho^{2} .
$$


It then follows from the proof of Theorem 2.1 that

$$
\frac{\operatorname{Vol}\left(U_{\rho}\left(C_{4}\right) \cap P(R)\right)}{\operatorname{Vol}(P(R))} \leq \frac{\rho^{2}}{R^{2}} .
$$

This proves Proposition 3.1.

Remark 3.2. As $C_{4} \subset \mathcal{W}_{4}, a_{3}^{3}-4 a_{3} a_{2}+8 a_{1}=0$ implies that $\Psi\left(a_{1}, a_{2}, a_{3}\right)=0$. So there should be a certain relation between $a_{3}^{3}-4 a_{3} a_{2}+8 a_{1}$ and $\Psi$. In fact, recall that

$$
\begin{aligned}
\Psi\left(a_{1}, a_{2}, a_{3}\right)=- & 4096\left(a_{3}^{3}-4 a_{3} a_{2}+8 a_{1}\right)^{2} \\
& \times\left(108 a_{1}^{2}-108 a_{3} a_{2} a_{1}+27 a_{3}^{3} a_{1}+32 a_{2}^{3}-9 a_{3}^{2} a_{2}^{2}\right)^{3} .
\end{aligned}
$$

It follows that $a_{3}^{3}-4 a_{3} a_{2}+8 a_{1}$ is a factor of $\Psi$ with multiplicity two.

3.2. Degree six polynomials. By considering composite polynomials of degree six, we also see that the density estimate of $C_{6}$ in Theorem 2.1 is not sharp. In fact, by Theorem 2.1,

$$
\frac{\operatorname{Vol}\left(U_{\rho}\left(C_{6}\right) \cap P(R)\right)}{\operatorname{Vol}(P(R))} \leq \frac{24 \rho^{2}}{R^{2}} .
$$

However, we actually have the following proposition which gives a better upper bound for the density of $C_{6}$.

Proposition 3.3.

$$
\frac{\operatorname{Vol}\left(U_{\rho}\left(C_{6}\right) \cap P(R)\right)}{\operatorname{Vol}(P(R))} \leq \frac{2 \rho^{2}}{R^{2}} .
$$

To see this, note that if $p(z)=z^{6}+a_{5} z^{5}+a_{4} z^{4}+a_{3} z^{3}+a_{2} z^{2}+a_{1} z$ is composite, then $\Psi\left(a_{1}, a_{2}, a_{3}, a_{4}, a_{5}\right)=0$. By using mathematical software such as Mathematica, $\Psi$ can be factorized to the following form:

$$
\Psi\left(a_{1}, a_{2}, a_{3}, a_{4}, a_{5}\right)=C\left[q\left(a_{1}, a_{2}, a_{3}, a_{4}, a_{5}\right)\right]^{3}\left[r\left(a_{1}, a_{2}, a_{3}, a_{4}, a_{5}\right)\right]^{2},
$$

for some constant $C$ and some polynomials $q, r \in \mathbb{C}\left[a_{1}, a_{2}, a_{3}, a_{4}, a_{5}\right]$ such that $q$ has degree four in $a_{1}$ and $r$ has degree six in $a_{1}$. From the proof of Theorem 2.1, we know that the upper bound $24 \rho^{2} / R^{2}$ comes from the fact that $\Psi\left(a_{1}, a_{2}, a_{3}\right)$ is of degree 24 in $a_{1}$. We can get a much better bound $2 \rho^{2} / R^{2}$ by showing the following lemma.

Lemma 3.4. The polynomial $p(z)=z^{6}+a_{5} z^{5}+a_{4} z^{4}+a_{3} z^{3}+a_{2} z^{2}+a_{1} z$ is composite if and only if

$$
\left\{\begin{array}{l}
5 a_{5}^{3}+27 a_{3}-18 a_{5} a_{4}=0 \\
a_{5}^{5}-3 a_{5}^{3} a_{4}+27 a_{5} a_{2}-81 a_{1}=0
\end{array}\right.
$$

or

$$
\left\{\begin{array}{l}
a_{5}^{5}-8 a_{5}^{3} a_{4}+8 a_{5}^{2} a_{3}+16 a_{5} a_{4}^{2}-32 a_{4} a_{3}+64 a_{1}=0 \\
5 a_{5}^{4}-24 a_{5}^{2} a_{4}+32 a_{5} a_{3}+16 a_{4}^{2}-64 a_{2}=0 .
\end{array}\right.
$$


Proof of Lemma 3.4. Suppose that $p(z)=z^{6}+a_{5} z^{5}+a_{4} z^{4}+a_{3} z^{3}+a_{2} z^{2}+a_{1} z$ is composite. Without loss of generality, we only need to consider the following two different kinds of factorizations:

(1) $z^{6}+a_{5} z^{5}+a_{4} z^{4}+a_{3} z^{3}+a_{2} z^{2}+a_{1} z=\left(z^{3}+A z^{2}+B z\right) \circ\left(z^{2}+C z\right)$;

(2) $z^{6}+a_{5} z^{5}+a_{4} z^{4}+a_{3} z^{3}+a_{2} z^{2}+a_{1} z=\left(z^{2}+A z\right) \circ\left(z^{3}+B z^{2}+C z\right)$.

For case 1,

$$
\begin{aligned}
& z^{6}+a_{5} z^{5}+a_{4} z^{4}+a_{3} z^{3}+a_{2} z^{2}+a_{1} z \\
& =z^{6}+3 C z^{5}+\left(3 C^{2}+A\right) z^{4}+\left(C^{3}+2 A C\right) z^{3}+\left(A C^{2}+B\right) z^{2}+B C z
\end{aligned}
$$

Comparing the coefficients,

$$
\left\{\begin{array}{l}
a_{5}=3 C \\
a_{4}=3 C^{2}+A \\
a_{3}=C^{3}+2 A C \\
a_{2}=A C^{2}+B \\
a_{1}=B C .
\end{array}\right.
$$

After eliminations, we obtain two equations $5 a_{5}^{3}+27 a_{3}-18 a_{5} a_{4}=0$ and $a_{5}^{5}-3 a_{5}^{3} a_{4}+$ $27 a_{5} a_{2}-81 a_{1}=0$.

Conversely, suppose that $5 a_{5}^{3}+27 a_{3}-18 a_{5} a_{4}=0$ and $a_{5}^{5}-3 a_{5}^{3} a_{4}+27 a_{5} a_{2}-$ $81 a_{1}=0$. Then

$$
\begin{aligned}
\left(z^{3}+\right. & \left.\left(a_{4}-\frac{a_{5}^{2}}{3}\right) z^{2}+\left(a_{2}-\frac{a_{5}^{2} a_{4}}{9}+\frac{a_{5}^{4}}{27}\right) z\right) \circ\left(z^{2}+\frac{a_{5}}{3} z\right) \\
& =z^{6}+a_{5} z^{5}+a_{4} z^{4}+\left(-\frac{5 a_{5}^{3}}{27}+\frac{2 a_{5} a_{4}}{3}\right) z^{3}+a_{2} z^{2}+\left(\frac{a_{5}^{5}}{81}-\frac{a_{5}^{3} a_{4}}{27}+\frac{a_{5} a_{2}}{3}\right) z \\
& =z^{6}+a_{5} z^{5}+a_{4} z^{4}+a_{3} z^{3}+a_{2} z^{2}+a_{1} z .
\end{aligned}
$$

So $p$ is composite.

For case 2,

$$
\begin{aligned}
z^{6}+ & a_{5} z^{5}+a_{4} z^{4}+a_{3} z^{3}+a_{2} z^{2}+a_{1} z \\
& =z^{6}+2 B z^{5}+\left(2 C+B^{2}\right) z^{4}+(A+2 B C) z^{3}+\left(C^{2}+A B\right) z^{2}+A C z .
\end{aligned}
$$

Comparing the coefficients,

$$
\left\{\begin{array}{l}
a_{5}=2 B \\
a_{4}=2 C+B^{2} \\
a_{3}=A+2 B C \\
a_{2}=C^{2}+A B \\
a_{1}=A C .
\end{array}\right.
$$

After eliminations, we obtain two equations $a_{5}^{5}-8 a_{5}^{3} a_{4}+8 a_{5}^{2} a_{3}+16 a_{5} a_{4}^{2}-32 a_{4} a_{3}+$ $64 a_{1}=0$ and $5 a_{5}^{4}-24 a_{5}^{2} a_{4}+32 a_{5} a_{3}+16 a_{4}^{2}-64 a_{2}=0$. 
Conversely, suppose that $a_{5}^{5}-8 a_{5}^{3} a_{4}+8 a_{5}^{2} a_{3}+16 a_{5} a_{4}^{2}-32 a_{4} a_{3}+64 a_{1}=0$ and $5 a_{5}^{4}-24 a_{5}^{2} a_{4}+32 a_{5} a_{3}+16 a_{4}^{2}-64 a_{2}=0$. Then

$$
\begin{aligned}
\left(z^{2}+\right. & \left.\left(a_{3}-\frac{a_{5} a_{4}}{2}+\frac{a_{5}^{3}}{8}\right) z\right) \circ\left(z^{3}+\frac{a_{5}}{2} z^{2}+\left(\frac{a_{4}}{2}-\frac{a_{5}^{2}}{8}\right) z\right) \\
= & z^{6}+a_{5} z^{5}+a_{4} z^{4}+a_{3} z^{3}+\left(\frac{5 a_{5}^{4}}{64}-\frac{3 a_{5}^{2} a_{4}}{8}+\frac{a_{4}^{2}}{4}+\frac{a_{5} a_{3}}{2}\right) z^{2} \\
& +\left(-\frac{a_{5}^{5}}{64}+\frac{a_{5}^{3} a_{4}}{8}-\frac{a_{5} a_{4}^{2}}{4}-\frac{a_{5}^{2} a_{3}}{8}+\frac{a_{4} a_{3}}{2}\right) z \\
= & z^{6}+a_{5} z^{5}+a_{4} z^{4}+a_{3} z^{3}+a_{2} z^{2}+a_{1} z
\end{aligned}
$$

So $p$ is composite.

Let

$$
\begin{aligned}
& R_{1}^{(1)}\left(a_{1}, a_{2}, a_{3}, a_{4}, a_{5}\right)=5 a_{5}^{3}+27 a_{3}-18 a_{5} a_{4}, \\
& R_{2}^{(1)}\left(a_{1}, a_{2}, a_{3}, a_{4}, a_{5}\right)=a_{5}^{5}-3 a_{5}^{3} a_{4}+27 a_{5} a_{2}-81 a_{1}, \\
& R_{1}^{(2)}\left(a_{1}, a_{2}, a_{3}, a_{4}, a_{5}\right)=a_{5}^{5}-8 a_{5}^{3} a_{4}+8 a_{5}^{2} a_{3}+16 a_{5} a_{4}^{2}-32 a_{4} a_{3}+64 a_{1}, \\
& R_{2}^{(2)}\left(a_{1}, a_{2}, a_{3}, a_{4}, a_{5}\right)=5 a_{5}^{4}-24 a_{5}^{2} a_{4}+32 a_{5} a_{3}+16 a_{4}^{2}-64 a_{2} .
\end{aligned}
$$

By Lemma 3.4,

$$
\begin{aligned}
C_{6}= & \left\{\left(a_{1}, a_{2}, a_{3}, a_{4}, a_{5}\right) \in \mathbb{C}^{5}: R_{1}^{(1)}=R_{2}^{(1)}=0 \text { or } R_{1}^{(2)}=R_{2}^{(2)}=0\right\} \\
= & \left\{\left(a_{1}, a_{2}, a_{3}, a_{4}, a_{5}\right) \in \mathbb{C}^{5}: R_{1}^{(1)}=R_{2}^{(1)}=0\right\} \\
& \cup\left\{\left(a_{1}, a_{2}, a_{3}, a_{4}, a_{5}\right) \in \mathbb{C}^{5}: R_{1}^{(2)}=R_{2}^{(2)}=0\right\} \\
= & C_{6}^{(1)} \cup C_{6}^{(2)},
\end{aligned}
$$

where $C_{6}^{(1)}=\left\{\left(a_{1}, a_{2}, a_{3}, a_{4}, a_{5}\right) \in \mathbb{C}^{5}: R_{1}^{(1)}=R_{2}^{(1)}=0\right\}$ and $C_{6}^{(2)}=\left\{\left(a_{1}, a_{2}, a_{3}, a_{4}, a_{5}\right) \in\right.$ $\left.\mathbb{C}^{5}: R_{1}^{(2)}=R_{2}^{(2)}=0\right\}$.

Now we look at some examples of composite polynomials of degree six.

(1) Let $p(z)=z^{6}+2 z^{4}+z^{2}$. It is easily seen that $(0,1,0,2,0) \in \mathbb{C}^{5}$ satisfies both (3.1) and (3.2). Hence $(0,1,0,2,0) \in C_{6}^{(1)} \cap C_{6}^{(2)} \subset C_{6}$. Therefore, there are two different kinds of factorizations for $p$ :

$$
z^{6}+2 z^{4}+z^{2}=\left(z^{3}+2 z^{2}+z\right) \circ z^{2}=z^{2} \circ\left(z^{3}+z\right) .
$$

(2) Let $p(z)=z^{6}+z^{4}+z^{2}$. It is easy to check that $(0,1,0,1,0) \in \mathbb{C}^{5}$ satisfies (3.1), but not (3.2). Hence $(0,1,0,1,0) \in C_{6}^{(1)} \backslash C_{6}^{(2)} \subset C_{6}$. Therefore,

$$
z^{6}+z^{4}+z^{2}=\left(z^{3}+z^{2}+z\right) \circ z^{2}
$$

and $z^{6}+z^{4}+z^{2}$ cannot be written in the form $\left(z^{2}+A z\right) \circ\left(z^{3}+B z^{2}+C z\right)$. 
(3) Let $p(z)=z^{6}+2 z^{4}+z^{3}+z^{2}+z$. It is easy to verify that $(1,1,1,2,0) \in \mathbb{C}^{5}$ satisfies (3.2), but not (3.1). Hence $(1,1,1,2,0) \in C_{6}^{(2)} \backslash C_{6}^{(1)} \subset C_{6}$. Therefore,

$$
z^{6}+2 z^{4}+z^{3}+z^{2}+z=\left(z^{2}+z\right) \circ\left(z^{3}+z\right)
$$

and $z^{6}+2 z^{4}+z^{3}+z^{2}+z$ cannot be written in the form $\left(z^{3}+A z^{2}+B z\right) \circ$ $\left(z^{2}+C z\right)$.

From the above examples, we have $C_{6}=C_{6}^{(1)} \cup C_{6}^{(2)}$, where $C_{6}^{(1)} \cap C_{6}^{(2)} \neq \emptyset, C_{6}^{(1)} \backslash C_{6}^{(2)}$ $\neq \emptyset$ and $C_{6}^{(2)} \backslash C_{6}^{(1)} \neq \emptyset$.

Proof of Proposition 3.3. It follows from (3.1) and (3.2) in Lemma 3.4 that for any $\left(a_{2}, a_{3}, a_{4}, a_{5}\right) \in \mathbb{C}^{4}$, there exists at most one $a_{1}^{\prime} \in \mathbb{C}$ such that

$$
\left(a_{1}^{\prime}, a_{2}, a_{3}, a_{4}, a_{5}\right) \in C_{6}^{(1)},
$$

and similarly there exists at most one $a_{1}^{\prime \prime} \in \mathbb{C}$ such that

$$
\left(a_{1}^{\prime \prime}, a_{2}, a_{3}, a_{4}, a_{5}\right) \in C_{6}^{(2)} .
$$

Therefore, the intersection of $C_{6}$ with the one-dimensional coordinate plane

$$
\left\{\left(z, a_{2}, a_{3}, a_{4}, a_{5}\right): z \in \mathbb{C}\right\}
$$

consists of at most two points. Hence

$$
\left|\int_{\left|a_{1}\right|<R} \chi\left(a_{1}, a_{2}, a_{3}, a_{4}, a_{5}\right) d a_{1}\right| \leq\left|\int_{\left|a_{1}\right|<\infty} \chi\left(a_{1}, a_{2}, a_{3}, a_{4}, a_{5}\right) d a_{1}\right| \leq 2 \pi \rho^{2} .
$$

It then follows from the proof of Theorem 2.1 that

$$
\frac{\operatorname{Vol}\left(U_{\rho}\left(C_{6}\right) \cap P(R)\right)}{\operatorname{Vol}(P(R))} \leq \frac{2 \rho^{2}}{R^{2}} .
$$

This proves Proposition 3.3.

Remark 3.5. As $C_{6} \subset \mathcal{W}_{6}, R_{1}^{(1)}=R_{2}^{(1)}=0$ or $R_{1}^{(2)}=R_{2}^{(2)}=0$ implies that $\Psi=0$. So there should be a certain relation between $R_{1}^{(1)}, R_{2}^{(1)}, R_{1}^{(2)}, R_{2}^{(2)}$ and $\Psi$. Now we discuss such relation. Recall that $\Psi$ can be factorized to the following form:

$$
\Psi\left(a_{1}, a_{2}, a_{3}, a_{4}, a_{5}\right)=C\left[q\left(a_{1}, a_{2}, a_{3}, a_{4}, a_{5}\right)\right]^{3}\left[r\left(a_{1}, a_{2}, a_{3}, a_{4}, a_{5}\right)\right]^{2},
$$

for some constant $C$ and some polynomials $q, r \in \mathbb{C}\left[a_{1}, a_{2}, a_{3}, a_{4}, a_{5}\right]$. Let $I^{(1)}$ and $I^{(2)}$ be the ideals $\left\langle R_{1}^{(1)}, R_{2}^{(1)}\right\rangle$ and $\left\langle R_{1}^{(2)}, R_{2}^{(2)}\right\rangle$ generated by $R_{1}^{(1)}, R_{2}^{(1)}$ and $R_{1}^{(2)}, R_{2}^{(2)}$ respectively. Using mathematical software such as Mathematica, we find a Groebner basis $G^{(1)}$ for $I^{(1)}$ and a Groebner basis $G^{(2)}$ for $I^{(2)}$. When dividing $r$ by $G^{(1)}$ and $G^{(2)}$ respectively, both the remainders are zero. Hence $r \in I^{(1)}$ and $r \in I^{(2)}$ or equivalently,

$$
r=r_{1}^{(1)} R_{1}^{(1)}+r_{2}^{(1)} R_{2}^{(1)}=r_{1}^{(2)} R_{1}^{(2)}+r_{2}^{(2)} R_{2}^{(2)}
$$

for some $r_{1}^{(1)}, r_{2}^{(1)}, r_{1}^{(2)}, r_{2}^{(2)} \in \mathbb{C}\left[a_{1}, a_{2}, a_{3}, a_{4}, a_{5}\right]$. Therefore,

$$
\Psi\left(a_{1}, a_{2}, a_{3}, a_{4}, a_{5}\right)=C q^{3}\left(r_{1}^{(1)} R_{1}^{(1)}+r_{2}^{(1)} R_{2}^{(1)}\right)^{2}=C q^{3}\left(r_{1}^{(2)} R_{1}^{(2)}+r_{2}^{(2)} R_{2}^{(2)}\right)^{2} .
$$




\section{References}

[1] A. F. Beardon, 'Composition factors of polynomials', Complex Var. Theory Appl. 43 (2001), 225-239.

[2] A. F. Beardon and T. W. Ng, 'On Ritt's factorization of polynomials', J. Lond. Math. Soc. (2) 62 (2000), 127-138.

[3] A. F. Beardon and T. W. Ng, 'Parametrizations of algebraic curves', Ann. Acad. Sci. Fenn. Math. 31 (2006), 541-554.

[4] C. T. Chuang and C. C. Yang, Factorization Theory and Fixed Points of Meromorphic Functions (World Scientific, Singapore, 1990).

[5] F. Dorey and G. Whaples, 'Prime and composite polynomials', J. Algebra 28 (1974), 88-101.

[6] H. T. Engstrom, 'Polynomial substitutions', Amer. J. Math. 63 (1941), 249-255.

[7] F. Gross, Factorization of Meromorphic Functions (Mathematics Research Center, Naval Research Laboratory, Washington, DC, 1972).

[8] H. Levi, 'Composite polynomials with coefficients in an arbitrary field of characteristic zero', Amer. J. Math. 64 (1942), 389-400.

[9] P. Müller, 'Primitive monodromy groups of polynomials', Contemp. Math. 186 (1995), 385-401.

[10] T. W. Ng and M. X. Wang, 'Ritt's theory on the unit disk', Forum Math. (2012).

[11] F. Pakovich, 'Prime and composite Laurent polynomials', Bull. Sci. Math. 133 (2009), 693-732.

[12] Q. I. Rahman and G. Schmeisser, Analytic Theory of Polynomials (Clarendon Press, Oxford, 2002).

[13] J. F. Ritt, 'Prime and composite polynomials', Trans. Amer. Math. Soc. (1) 23 (1922), 51-66.

[14] S. Smale, 'The fundamental theorem of algebra and complexity theory', Bull. Amer. Math. Soc. (N.S.) 4 (1981), 1-36.

[15] H. Urabe, 'On factorization of the Blaschke products', Bull. Kyoto Univ. Ed. Ser. B 63 (1983), $1-13$.

[16] U. Zannier, 'Ritt's second theorem in arbitrary characteristic', J. reine angew. Math. 445 (1993), 175-203.

\section{WAI SHUN CHEUNG, Department of Mathematics, The University of Hong Kong, Pokfulam, Hong Kong e-mail: cheungwaishun@gmail.com}

TUEN WAI NG, Department of Mathematics, The University of Hong Kong, Pokfulam, Hong Kong e-mail:ntw@maths.hku.hk

CHIU YIN TSANG, Department of Mathematics, The University of Hong Kong, Pokfulam, Hong Kong e-mail: ssuperhk@yahoo.com.hk 